\title{
Towards the Development of Effective Video Segmentation Based on Skew Gaussian Mixture Model
}

\author{
Pavan Kumar Tadiparthi, Srinivas Yarramalle
}

\begin{abstract}
: video analysis has gained a exponential demand with its usage in security cameras and in most of the real time applications for monitoring the law order. In order to have a precise analysis background subtraction and foreground detection processed are generally considered in the most of the approaches. However, to have a more precise output from the dynamic motion images, this article proposes a methodology based on skew Gaussian mixture model. The results are analyzed against the existing methods using quality assessment measures.
\end{abstract}

Index terms: Performance analysis; image segmentation; skew Gaussian; Background subtraction; quality metrics.

\section{INTRODUCTION}

In most of the image analysis techniques highlighted in case of video sequences, background subtraction is considered to be a crucial element. This Background subtraction helps to identify the reference background and there by assist to identify the movement objects using the disparity among the background an input image. Many methodologies uses the concept of both model and non-model based approaches of which majority of the articles are mostly focusing on extracting the regions, contours and also some recent articles have been proposed by the author in this direction using Generalized Rayleigh Distribution [1].The above article highlights about a methodology for effective identification of foreground images using the GRD methodology proposed .In same practical situations, the background methodologies used for subtraction from the foreground considers in particular cases of a temporarily halted objects and try to process the foreground detection. The results in these cases may not yield better results. background is to take into account the final details of the objects during time intervals ' $t$ ' and ' $t-1$ ', This consideration helps to generate better understanding about the image to be segmented also.

In some practical situations are may came across the images that are skewed and having highest kurtosis and these sort of images have a limitation for segmenting based on Generalized Rayleigh distribution (GRD). Therefore, to counter attack such variations in kurtosis, the present methodology based on skew Gaussian is considered.

Revised Manuscript Received on February 05, 2020.

* Correspondence Author

Pavan Kumar Tadiparthi, Associate Professor, Department of Information Technology, MVGR College of Engineering, India.

Srinivas Yarramalle, Professor, Department of Information Technology, GIT, GITAM University, India.

(C) The Authors. Published by Blue Eyes Intelligence Engineering and Sciences Publication (BEIESP). This is an open access article under the CC BY-NC-ND license (http://creativecommons.org/licenses/by-nc-nd/4.0/)
The most insightful methodology to consider the

The main limitation with respect to the Generalized Rayleigh Distribution (GRD) is that its considerations of low range sale factor ' $\alpha$ 'which is mostly equal to 0.5 and any image having scale above this can't be well interpreted. In contrary, the Skew Gaussian can consider the ranges up to plus or minus 3 and hence better perception can be obtained using this method. Therefore the present article aims at proposing the image segmentation methodology based on skew Gaussian.

The rest of the article is highlighted as follows, In section II, a brief overview of Skew Gaussian is presented, and section III highlights the considered data set. In section IV of the article presence the methodology, section $\mathrm{V}$ proposes the experimentation and Results derived together with performance is presented is corresponding section VI. The final section VII summarization the article with conclusion.

\section{FINITE SKEW NORMAL MIXTURE DISTRIBUTION}

Every image is a collection of several image regions. In each image region, the image data is quantized by pixel, which is a random variable because of the fact it is influenced by random factors like Vision, brightness, contrast etc. To model the pixel intensities in a image region, it is necessary to assume that the pixels in each image region follow a skew normal distribution. The probability density function of the pixel intensity is given by [Azzalini A. (1985), Tsung I Lin et al (2007)]

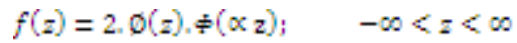

$$
\begin{aligned}
& \text { where, } \Phi(\propto \mathrm{z})=\int_{-\infty}^{\infty \mathrm{z}} \Phi(t) d t \\
& \text { and, } \quad \phi(z)=\frac{e^{-\frac{1}{z} z^{z}}}{\sqrt{2 \pi}} \\
& -3 \\
& \text { Let, } \quad y=\mu+\sigma z \\
& z=\frac{y-\mu}{\sigma}
\end{aligned}
$$

Substituting equations (2), (3), and (4) in equation (1),

$$
f(y)=\sqrt{\frac{2}{\pi}} \cdot e^{-\frac{1}{2}\left(\frac{y-\mu}{\sigma}\right)^{2}}\left[\int_{-\infty}^{\alpha\left(\frac{z-\mu}{\sigma}\right)} \frac{e^{-\frac{1}{2}\left(\frac{t-\mu}{\sigma}\right)^{2}}}{\sqrt{2 \pi}} d t\right]_{-5}
$$

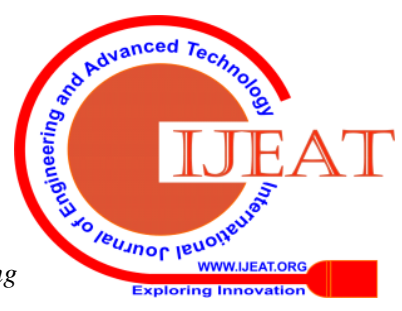


The mean pixel intensity of an image region is

$E(z)=\mu+\sqrt{\frac{2}{\pi}} . \delta(y) \quad-6$

Where,

$\delta(y)=\frac{\lambda}{\sqrt{1+\lambda^{2}}}$

The variance of the skew normal distribution is

$\operatorname{var}(z)=\left\{1-\frac{2}{\pi} \cdot \delta^{2}(\lambda)\right\} \cdot \sigma^{2}$

The moments of the skew normal distribution are given by,

$\mu=m_{1}-a_{1}\left(\frac{m_{3}}{b_{1}}\right)^{\frac{1}{3}}$
$\sigma^{2}=m_{2}-a_{1}^{2}\left(\frac{m_{\mathrm{g}}}{b_{1}}\right)^{\frac{a}{3}}$
$\delta(\lambda)=\left\{a_{1}{ }^{2}+m_{2}\left(\frac{b_{1}}{m_{3}}\right)^{\frac{2}{3}}\right\}^{-\frac{1}{2}}$ where,

$a_{1}=\sqrt{\frac{2}{\pi}} ; \quad b_{1}=\left(\frac{4}{\pi-1}\right) a_{1} ;$

$m_{1}=n^{-1} \sum_{i=1}^{n} y_{i}$

$m_{2}=(n-1)^{-1} \sum_{i=1}^{n}\left(y_{i}-\overline{y_{1}}\right)^{2}$

$m_{3}=(n-1)^{-1} \sum_{i=1}^{n}\left(y_{i}-\overline{y_{i}}\right)^{3}$

Since, the entire image is a collection of regions which are characterized by skew normal variants, we assume that the pixel intensities in the image region follows a k-component finite skew normal distribution and its probability density function is of the form

$h(x)=\sum_{i=1}^{k} \alpha_{i} g_{i}\left(y_{i} \mid \mu_{i}, \sigma_{i}^{2}, \lambda\right)$

here, $\lambda$ is the skewness parameter and $\mathrm{k}$ is the number of regions, $\alpha_{i}>0$ are weights such that $\sum_{i=1}^{k} \alpha_{i}=1$ and

$$
\begin{aligned}
& g\left(y_{i} \mid \mu_{i}, \sigma^{2}, \lambda\right)= \\
& \frac{2}{\pi} \cdot \frac{1}{\sqrt{2 \pi}} \cdot e^{-\frac{1}{2}\left(\frac{y-\mu}{\sigma}\right)^{2}} . \Phi\left(\lambda\left(\frac{y-\mu}{\sigma}\right)\right)
\end{aligned}
$$

where, $\Phi\left(\lambda\left(\frac{y-\mu}{\sigma}\right)\right)=\int_{-\infty}^{\lambda\left(\frac{y-\mu}{\sigma}\right)} \frac{1}{\sqrt{2 \pi}} \cdot e^{-\frac{t^{3}}{2}} d t$

$\mu_{i}, \sigma_{i}^{2}$ and $g_{i}\left(y_{i} \mid \theta\right)$, where $\theta=\left(\mu, \sigma^{2}, \lambda\right)$ are the mean, variance, and probability density function of the pixel in the $\mathrm{i}^{\text {th }}$ region respectively.

$\alpha_{i}$ is the probability of occurrence of the $i^{\text {th }}$ component of the finite skew normal mixture model, i.e., the probability of the $\mathrm{i}^{\text {th }}$ image region. Generally, it can be taken as the ratio of the size of the $i^{\text {th }}$ image region to the size of the entire image such that, $\sum_{\mathrm{i}=1}^{k} \alpha_{\mathrm{i}}=1$

\section{ESTIMATION OF MODEL PARAMETERS BY EM ALGORITHM}

In this section, we derive the updated equations of the model parameters using EM algorithm. The likelihood function of the observations $\mathrm{y}_{1}, \mathrm{y}_{2}, \mathrm{y}_{3} \ldots \ldots . \mathrm{y}_{\mathrm{n}}$ drawn from a image is given by

$L(\theta)=\prod_{\mid=1}^{d}\left(\sum_{i=1}^{k} \pi-n \cdot(Z A)\right) \quad-10$

Where,

$=\quad \theta=\left(\omega_{i}, \sigma_{\mathrm{i}}^{2}, \alpha_{\mathrm{i}}=1,2,3 \ldots \mathrm{k}\right)$ is the parameter set.
$\prod_{\mathrm{i}=1}^{M}\left(\sum_{\mathrm{i}=1}^{k} \alpha_{\mathrm{i}}\left[\frac{2}{\sigma} \cdot \frac{1}{\sqrt{2 \pi}} \cdot e^{-\frac{1}{2}\left(\frac{y-\mu}{\sigma}\right)^{2}} \Phi\left(\lambda\left(\frac{y-\mu}{\sigma}\right)\right)\right]\right)$
This implies.

$L(\theta)=$

$\sum_{z=1}^{N}\left[\sum_{i=1}^{k}\left[\prod_{i=1}^{N}\left(\sum_{i=1}^{k} \alpha_{i}\left[\frac{2}{\sigma} \cdot \frac{1}{\sqrt{2 \pi}} \cdot e^{-\frac{1}{n}\left(\frac{\gamma-\mu}{\sigma}\right)^{2}} \Phi\left(\lambda\left(\frac{y-\mu}{\sigma}\right)\right)\right]\right)\right]\right]$

where, $\Phi\left(\lambda\left(\frac{y-\mu}{\sigma}\right)\right)=\int_{-\infty}^{\lambda\left(\frac{y-\mu}{\sigma}\right)} \frac{1}{\sqrt{2 \pi}} \cdot e^{-\frac{t^{2}}{2}} d t$

The first step of EM algorithm requires the estimation of some reasonable initial estimates for both parameters $\theta^{(0)}$ and component weights $\alpha^{(0)}$ from the observed sample. The idea of EM algorithm is to evaluate the maximum likelihood estimates of the unknown parameter ${ }^{\prime} \theta^{\prime}$ by iterative process

\section{DATA SET}

In order to validate the efficacy of the developed algorithm, the methodology is proposed by taking the benchmark data set of CDnet 2014.

It consists of six categories of video frames ranging up to 80000.It also containing the frames pertaining to shadows, illumination effects.

\section{METHODOLOGY}

In this methodology each of the image is considered and the pixels are estimated into back ground and foreground images based on the threshold selected. 
The threshold is considered as a difference between the current frame and the previous frame. The pixels with high threshold values are given as input to the skew Gaussian model is presented in section II of the article. The probability density function (pdf) against each of the intensity values are given as input to the model and the respective values are estimated .These values which are below the threshold value are considered as background information else they are consider as foreground information.

\section{EXPERIMENTATION}

The experimentation carried out in mat lab environment using the bench mark data set 2014 and extracting the image background features in line with the proposed model based on skew Gaussian distribution.

The initial estimation of the parameters are estimated by using K-means algorithm and E.M algorithm is considered for further updating of these parameters. The segmentation is carried by maximizing the probability function of the skew Gaussian model is presented in the section II of the article. The results were also compared with the model based on Gaussian Mixture Model.

\section{PERFORMANCE EVALUATION AND EXPERIMENTAL RESULTS}

In order to validate the proposed model, we have considered the assessment metrics precision, Recall, Accuracy, F-Score, MSE, RMSE, FNR, FPR, PSNR, PWC The efficacy of the model can be justified by the value of computed precision ,if it is high ,it signifies high performance. On the other side, if method allocates the most of the pixels to background, the output precision value may be high, but proportionally the value of recall falls.

The formulas for computations of the above metrics are given by

Precision=TP $/(\mathrm{TP}+\mathrm{FP})$

Recall $=\mathrm{TP} /(\mathrm{TP}+\mathrm{FN})$

Accuracy $=\mathrm{TP}+\mathrm{TN} /(\mathrm{TP}+\mathrm{TN}+\mathrm{FP}+\mathrm{FN})-14$

F-score $=(2 *$ Precision $*$ recall $)$

(Precision +recall)

$\mathrm{MSE}=\mathrm{FP}+\mathrm{FN} / \mathrm{M} * \mathrm{~N}$

RMSE $=$ MSE

$\mathrm{FNR}=\mathrm{FN} /(\mathrm{TP}+\mathrm{FN})$

$\mathrm{FPR}=\mathrm{FP} /(\mathrm{FP}+\mathrm{TN})$

$\mathrm{PSNR}=10 \log _{10}\left(\mathrm{R}^{2} / \mathrm{MSE}\right)$

$\mathrm{PWC}=100 *(\mathrm{FN}+\mathrm{FP}) /(\mathrm{FN}+\mathrm{TN}+\mathrm{FP}+\mathrm{TP})$

The results derived using the proposed methodology is presented in the following tables 1 to 4 and the graphs based on assessment metrics in Fig .2 to 41.

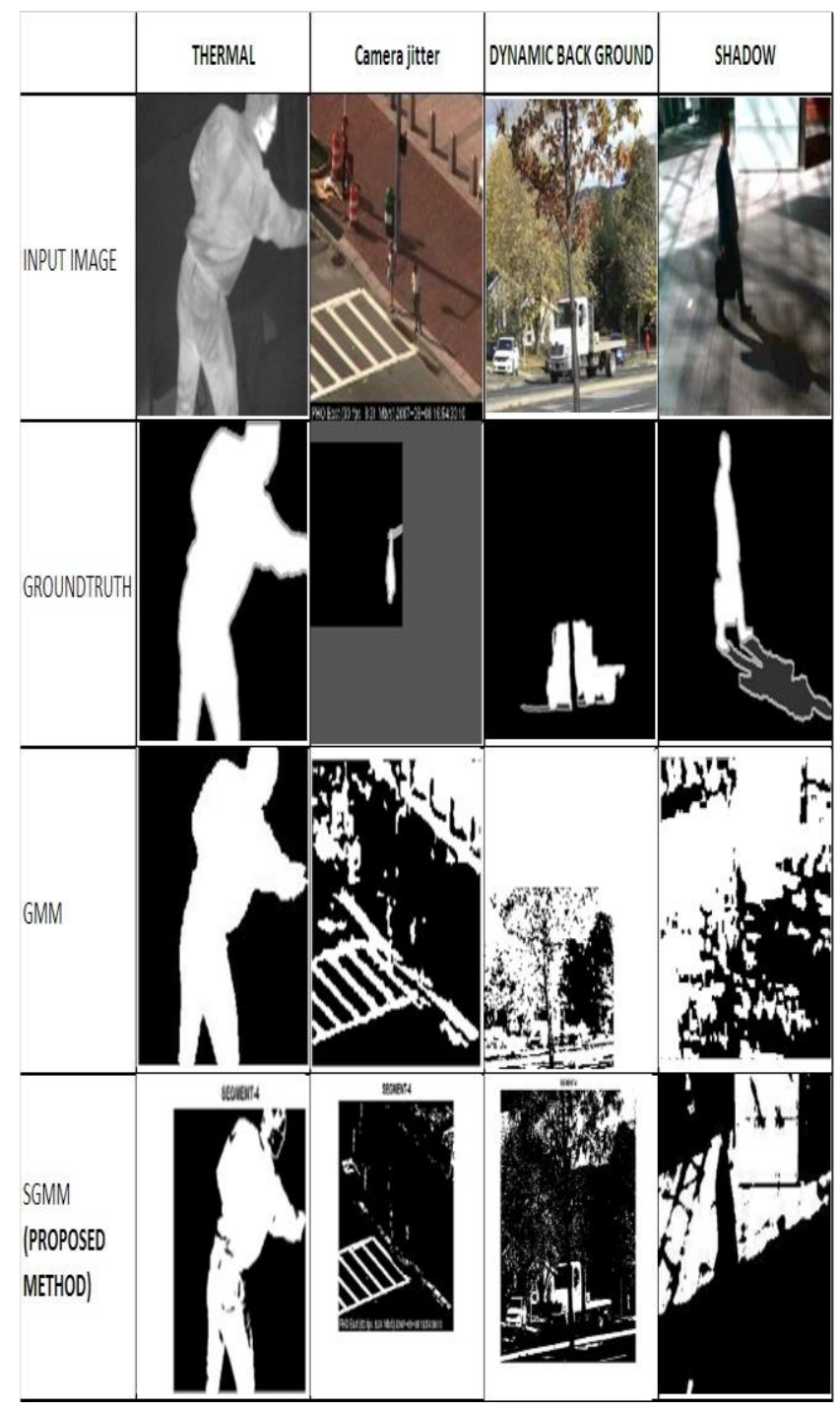

Fig1: Foreground detection of thermal, camera jitter, Dynamic background, Shadow from the CDNet2014 Dataset.

TABLE .1

Evaluation Metrics of different methods on THERMAL video from CD net DATASET

\begin{tabular}{|l|r|r|}
\hline $\begin{array}{c}\text { Metrics } \\
\text { Methods }\end{array}$ & GMM & \multicolumn{1}{c|}{ SGMM } \\
\hline PRECISION & 0.0238 & 0.0568 \\
\hline RECALL & 0.175 & 0.0237 \\
\hline ACCURACY & 0.9561 & 0.9912 \\
\hline F-SCORE & 0.0323 & 0.0615 \\
\hline MSE & 0.0152 & 0.0032 \\
\hline RMSE & 0.1212 & 0.0324 \\
\hline FPR & 0.0205 & 0.0040 \\
\hline FNR & 0.7032 & 0.8562 \\
\hline PSNR & 64.7802 & 77.826 \\
\hline PWC & 4.3823 & 2.8162 \\
\hline
\end{tabular}

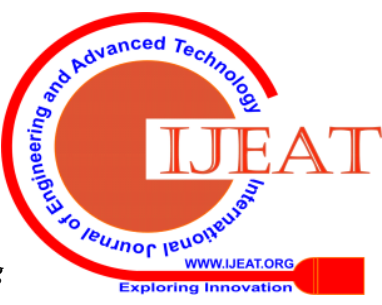


Towards the Development of Effective Video Segmentation Based on Skew Gaussian Mixture Model

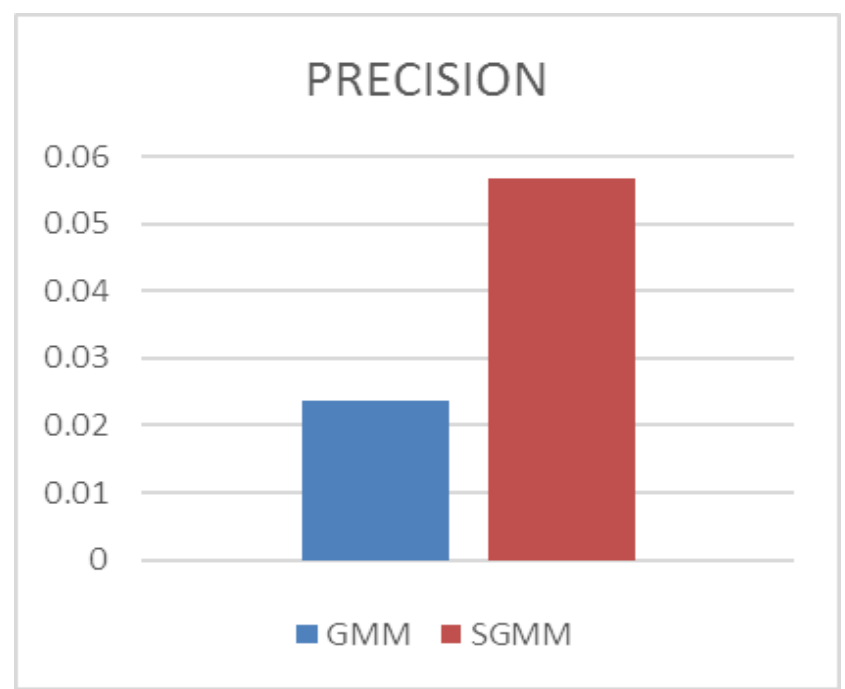

Fig.2 Precision of proposed method on Thermal dataset

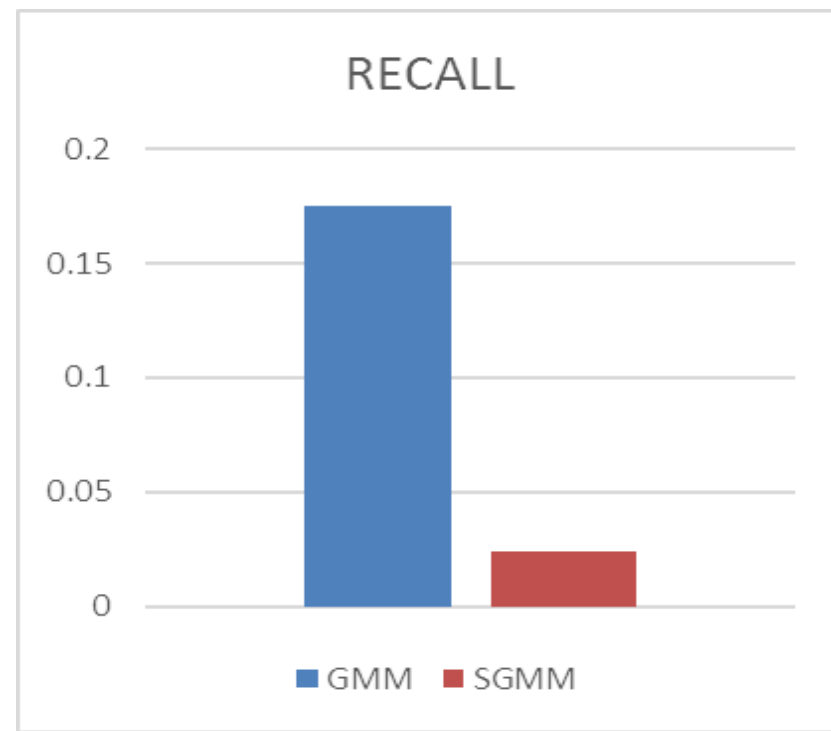

Fig.3 Recall of proposed method on Thermal dataset

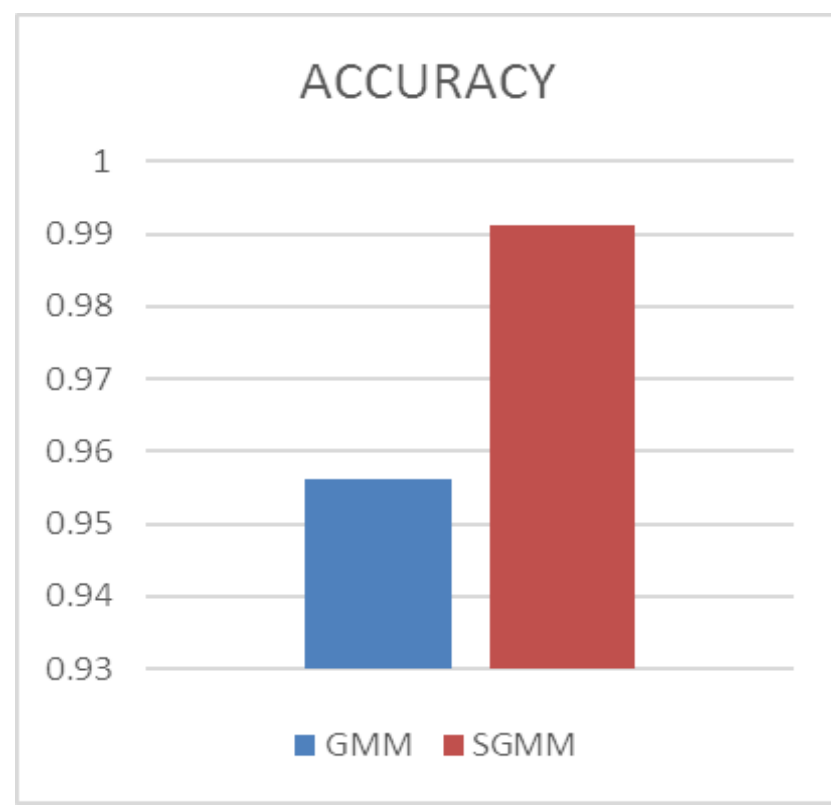

Fig.4 Accuracy of proposed method on Thermal dataset

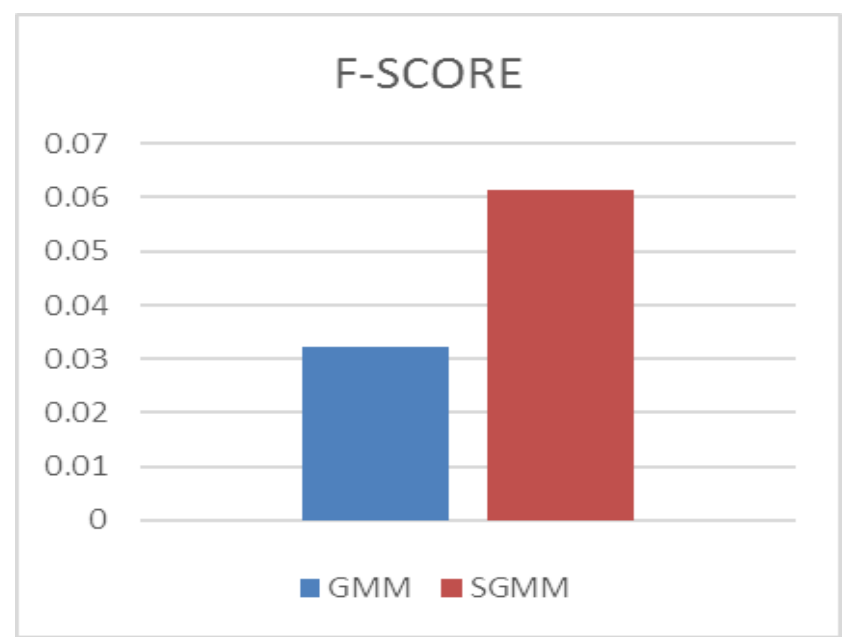

Fig.5 F-Score of proposed method on Thermal dataset

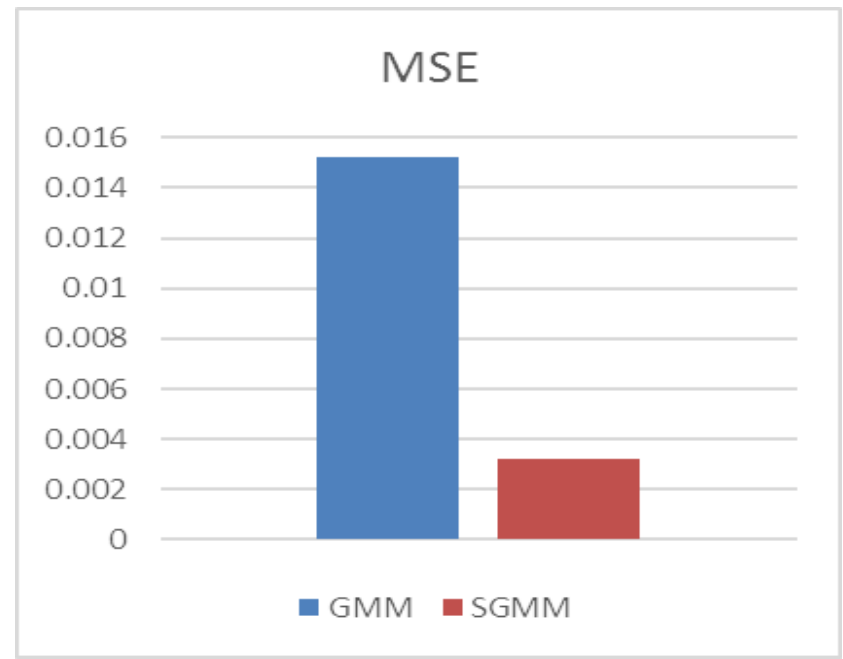

Fig.6 MSE of proposed method on Thermal dataset

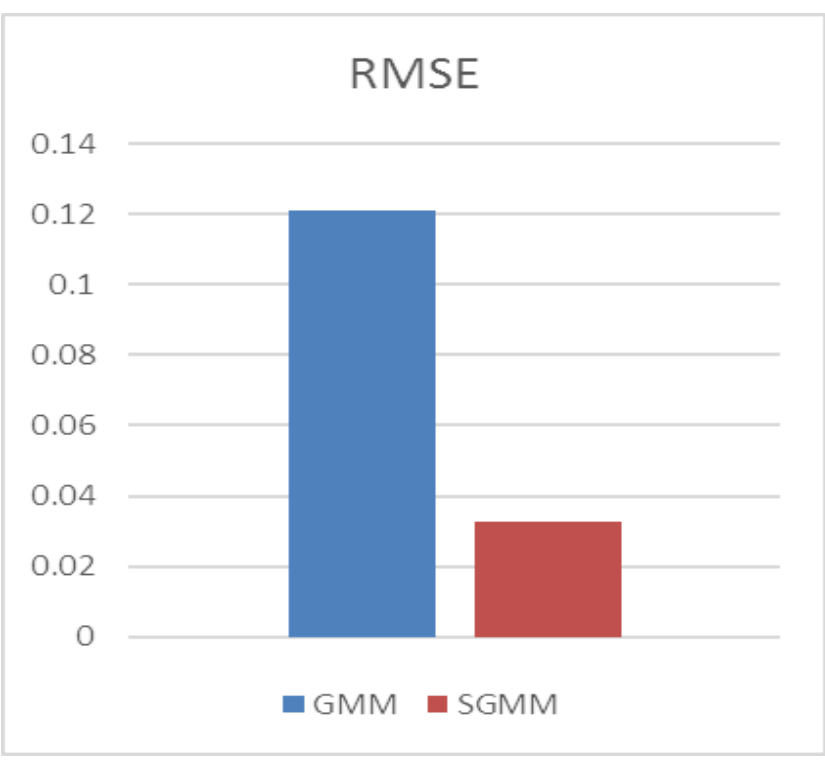

Fig.7 RMSE of proposed method on Thermal dataset

Published By:

1738 Blue Eyes Intelligence Engineering \& Sciences Publication 


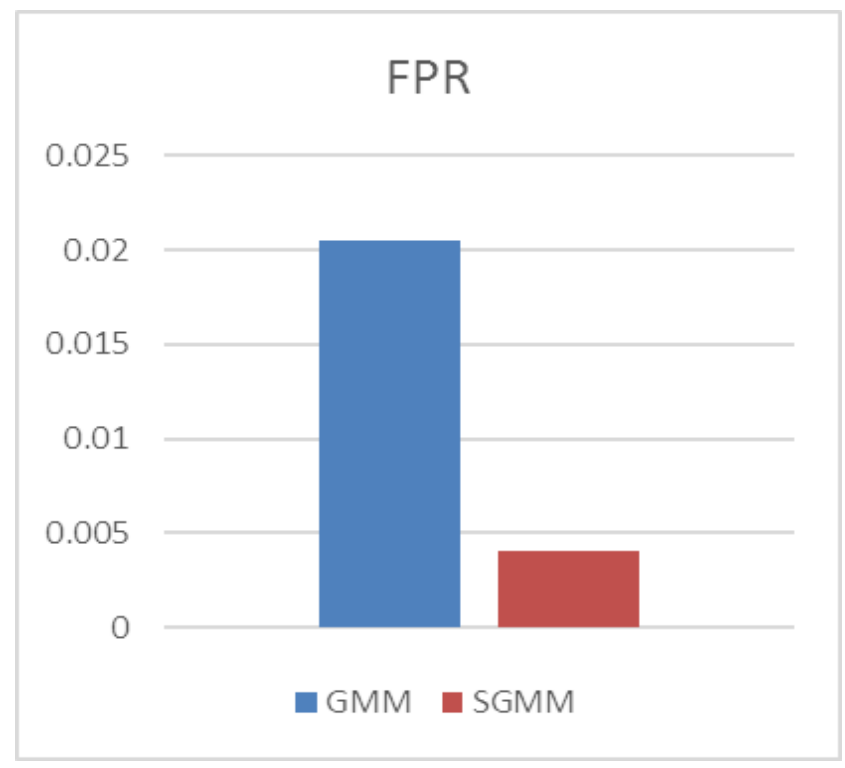

Fig.8 FPR of proposed method on Thermal dataset

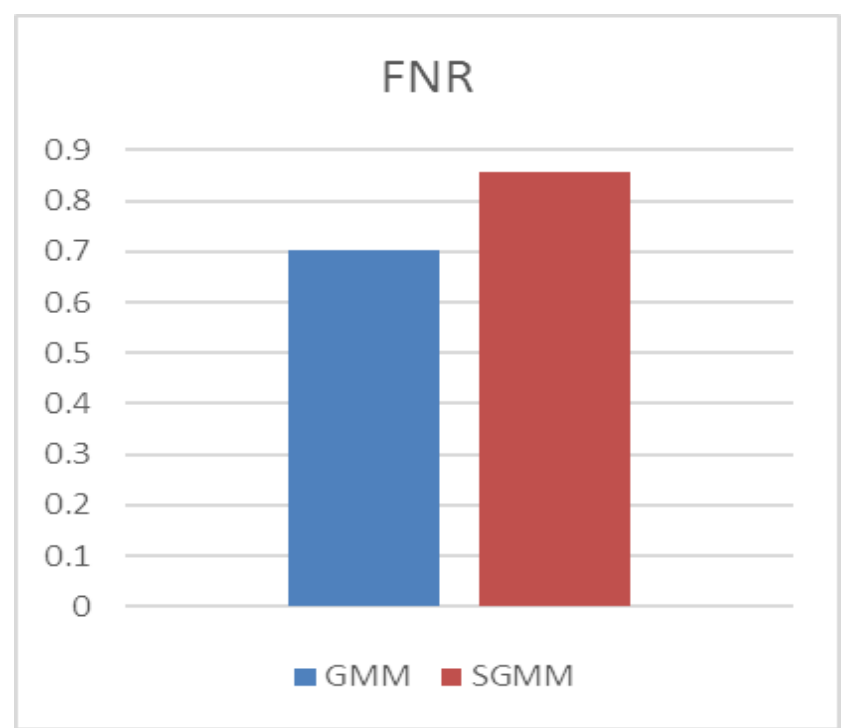

Fig.9 FNR of proposed method on Thermal dataset

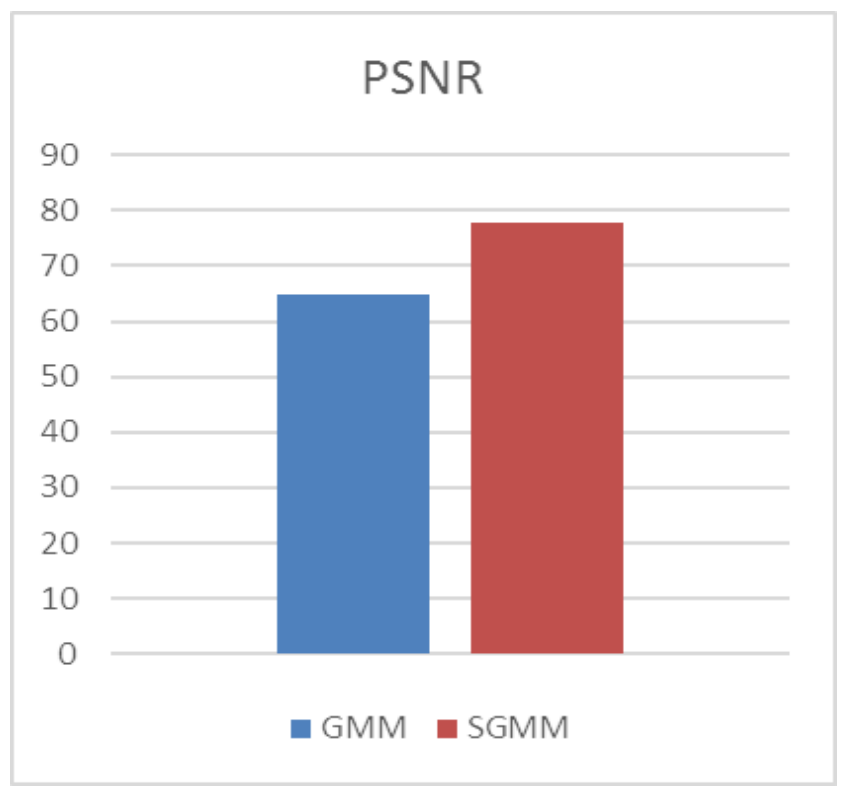

Fig.10 PSNR of proposed method on Thermal dataset

Retrieval Number: C5712029320/2020@BEIESP

DOI: 10.35940/ijeat.C5712.029320

Journal Website: www.ijeat.org

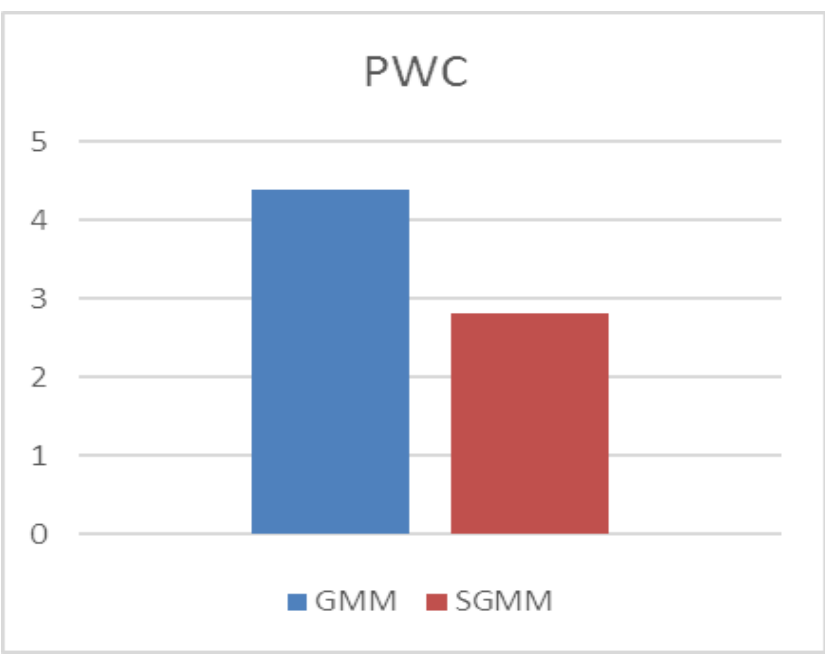

Fig.11 PWC of proposed method on Thermal dataset

TABLE .2

\begin{tabular}{|l|r|r|}
\hline \multicolumn{3}{|c|}{$\begin{array}{c}\text { Evaluation Metrics of different methods on } \\
\text { CAMERA JITTER video from CD net } \\
\text { DATASET }\end{array}$} \\
\hline $\begin{array}{c}\text { Metrics } \\
\text { Methods }\end{array}$ & GMM & \multicolumn{1}{c|}{ SGMM } \\
\hline PRECISION & 0.0346 & 0.0708 \\
\hline RECALL & 0.083 & 0.0572 \\
\hline ACCURACY & 0.9652 & 0.9953 \\
\hline F-SCORE & 0.0255 & 0.0532 \\
\hline MSE & 0.012 & 0.0232 \\
\hline RMSE & 0.0067 & 0.1435 \\
\hline FPR & 0.0208 & 0.0038 \\
\hline FNR & 0.805 & 0.9218 \\
\hline PSNR & 80.321 & 88.1032 \\
\hline PWC & 3.0483 & 1.6321 \\
\hline
\end{tabular}

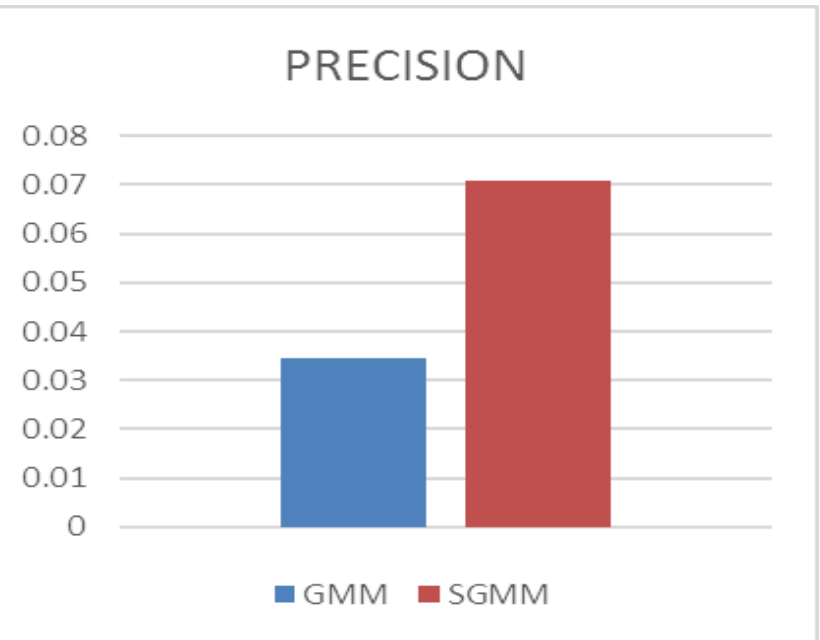

Fig.12 Precision of proposed method on Camera Jitter dataset 
Towards the Development of Effective Video Segmentation Based on Skew Gaussian Mixture Model

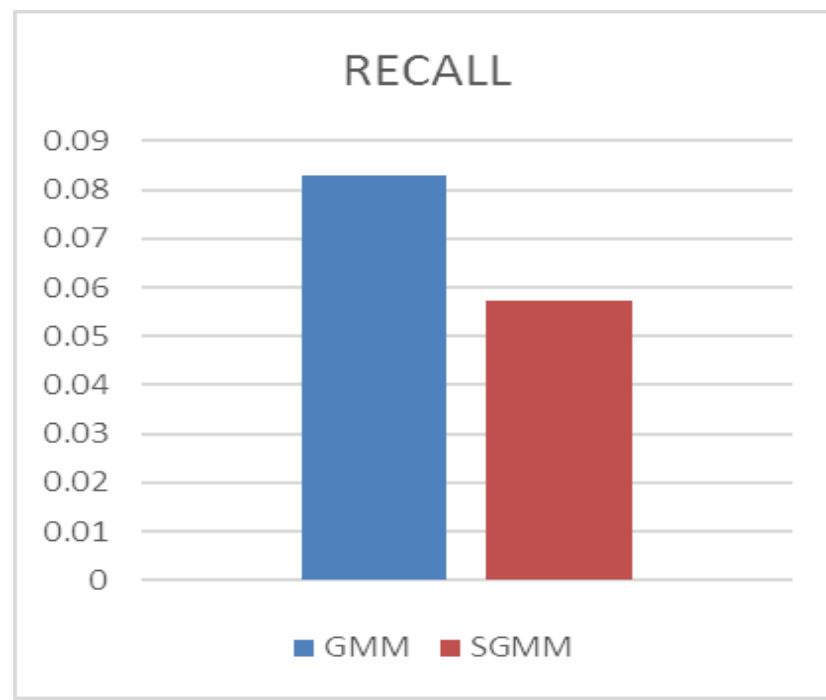

Fig.13 Recall of proposed method on Camera Jitter dataset

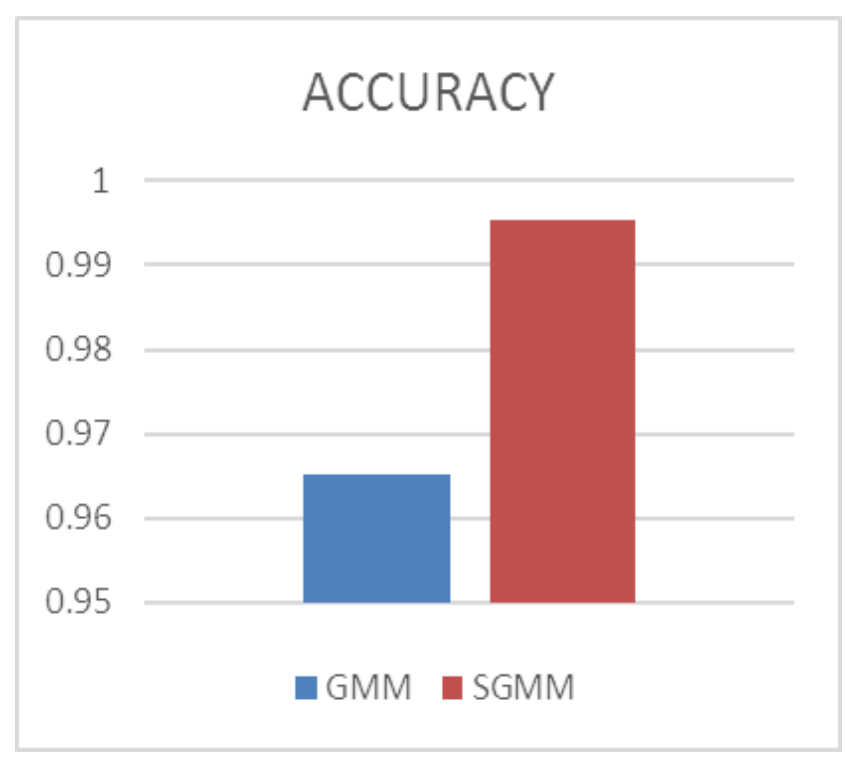

Fig.14 Accuracy of proposed method on Camera Jitter dataset

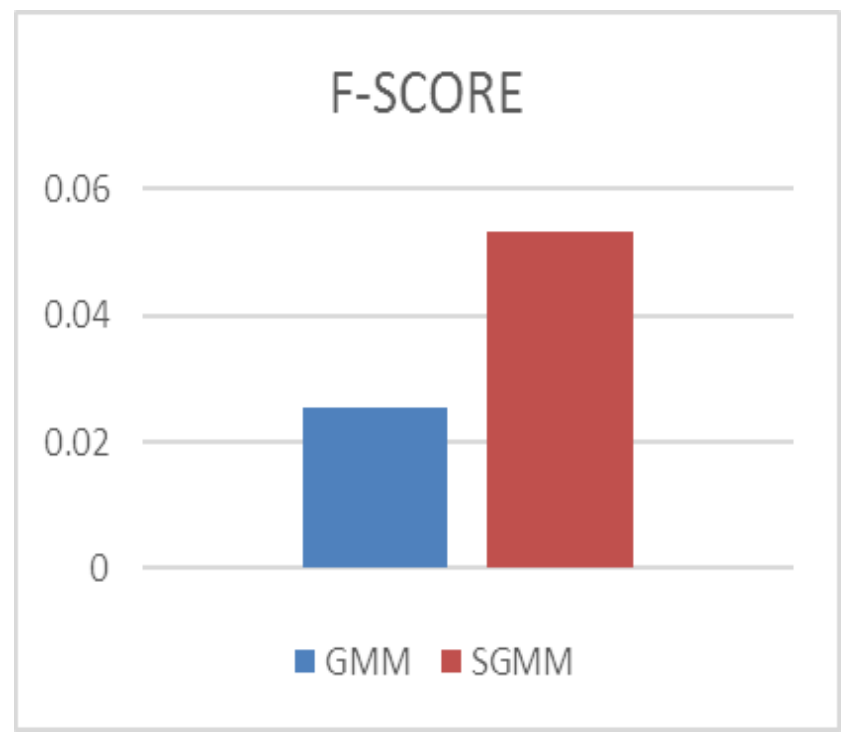

Fig.15 F-Score of proposed method on Camera Jitter dataset
Fig.16 MSE of proposed method on Camera Jitter dataset

\section{RMSE}

0.2

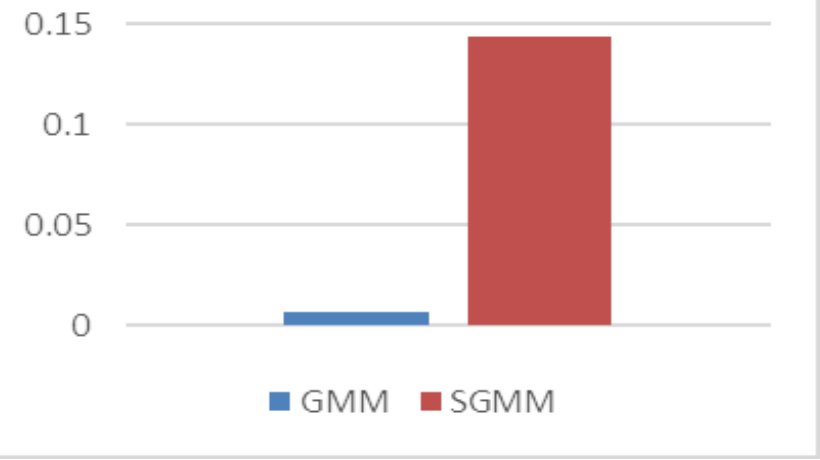

Fig.17 RMSE of proposed method on Camera Jitter dataset

\section{FPR}

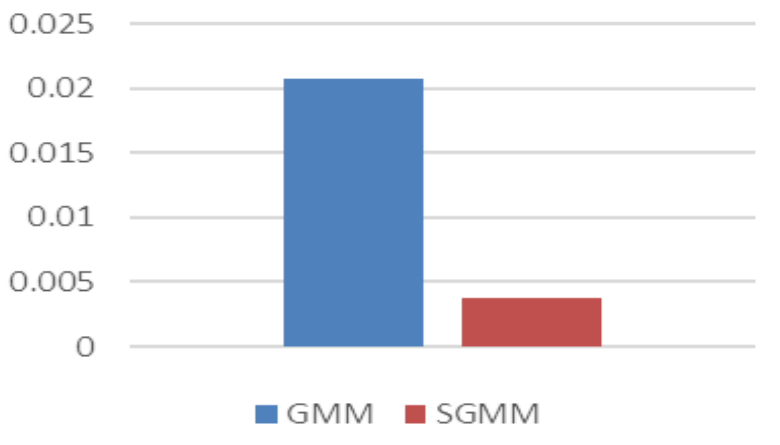

Fig.18 FPR of proposed method on Camera Jitter dataset

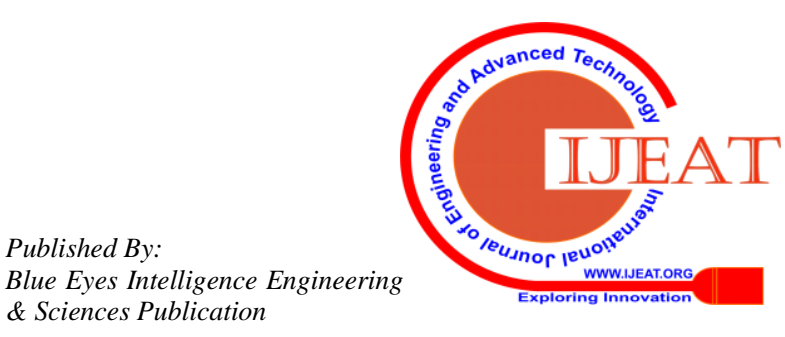




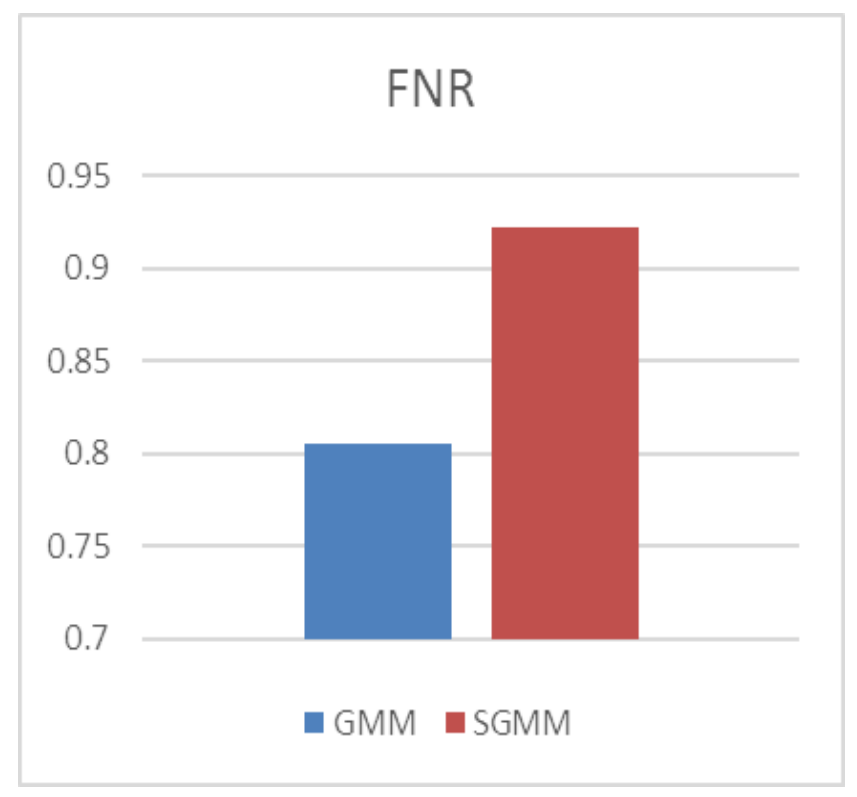

Fig.19 FNR of proposed method on Camera Jitter dataset

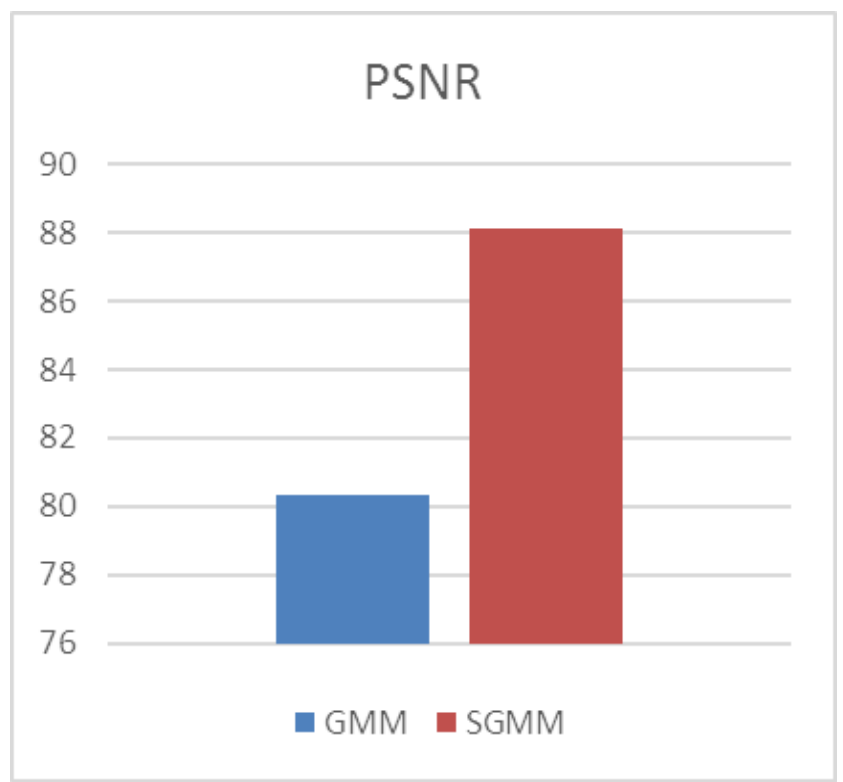

Fig.20 PSNR of proposed method on Camera Jitter dataset

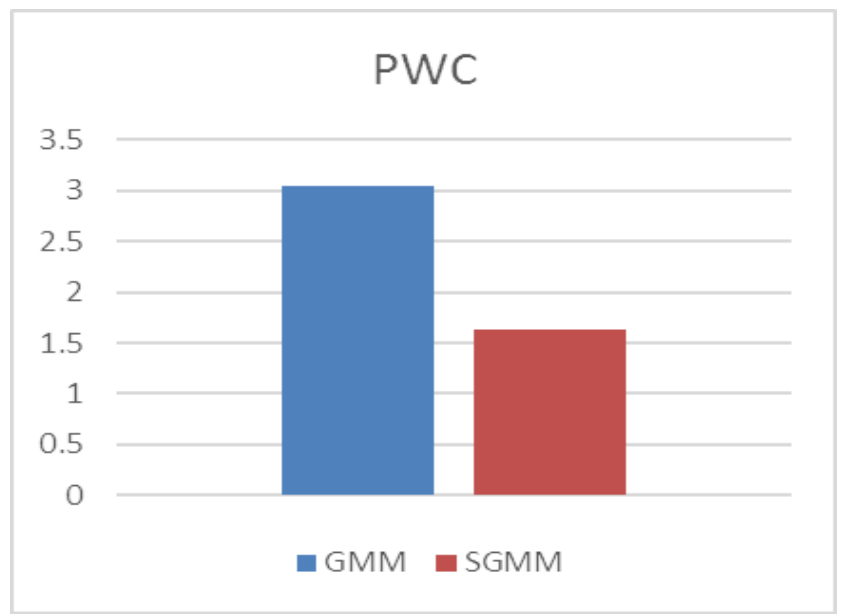

Fig.21 PWC of proposed method on Camera Jitter dataset

TABLE .3

Evaluation Metrics of different methods on dynamic back ground video from CD net DATASET

\begin{tabular}{|l|r|r|}
\hline \multicolumn{1}{|c|}{$\begin{array}{c}\text { Metrics } \\
\text { Methods }\end{array}$} & \multicolumn{1}{c|}{ GMM } & \multicolumn{1}{c|}{ SGMM } \\
\hline PRECISION & 0.012 & 0.0209 \\
\hline RECALL & 0.023 & 0.0128 \\
\hline ACCURACY & 0.9632 & 0.9932 \\
\hline F-SCORE & 0.017 & 0.0323 \\
\hline MSE & 0.003 & 0.0035 \\
\hline RMSE & 0.0161 & 0.0342 \\
\hline FPR & 0.023 & 0.0042 \\
\hline FNR & 0.032 & 0.8218 \\
\hline PSNR & 83.523 & 84.1021 \\
\hline PWC & 3.4325 & 2.6281 \\
\hline
\end{tabular}

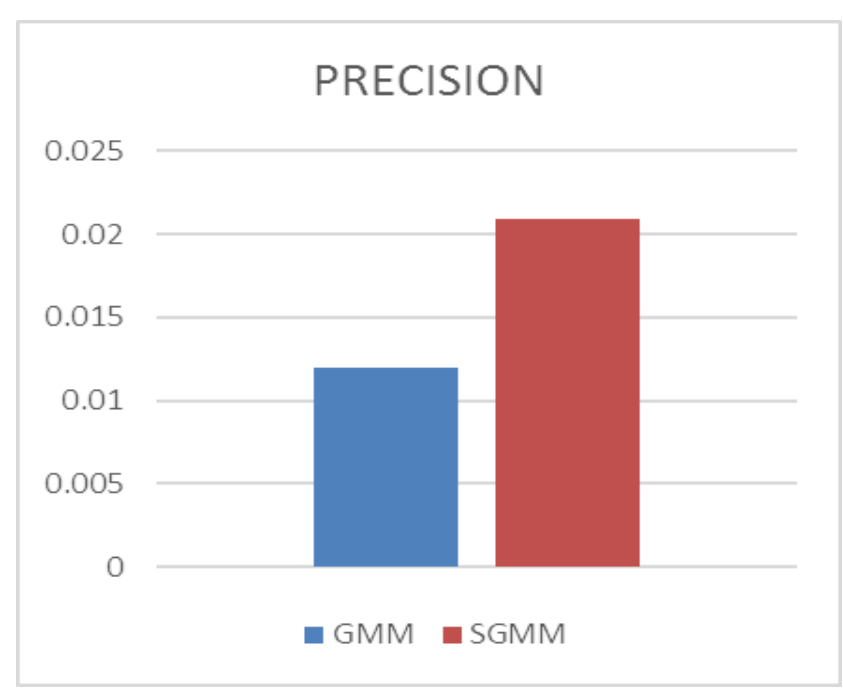

Fig.22 Precision of proposed method on dynamic back ground dataset

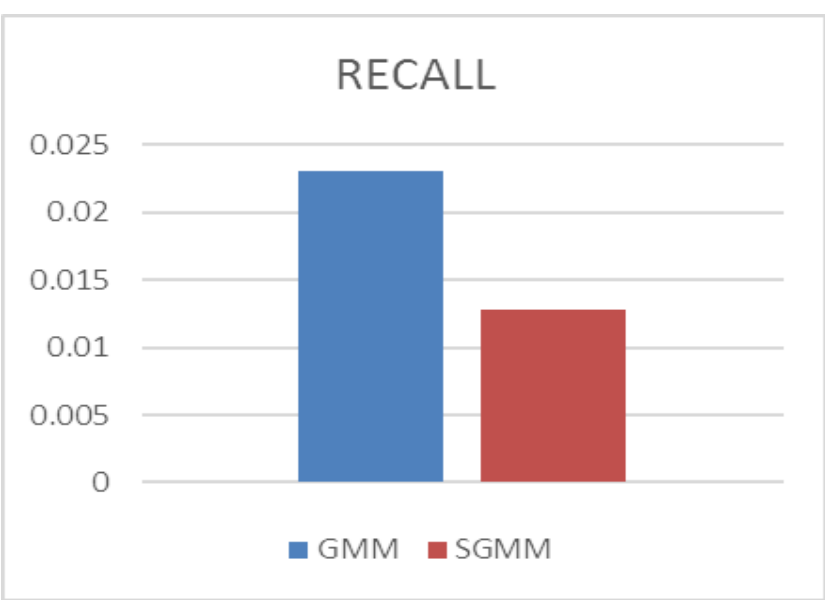

Fig.23 Recall of proposed method on dynamic back ground dataset 
Towards the Development of Effective Video Segmentation Based on Skew Gaussian Mixture Model

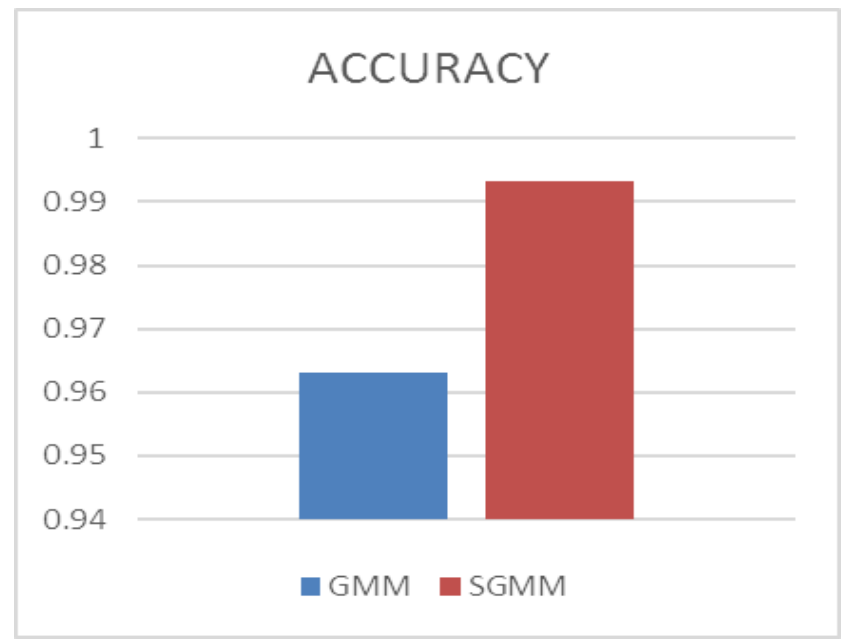

Fig.24 Accuracy of proposed method on dynamic back ground dataset

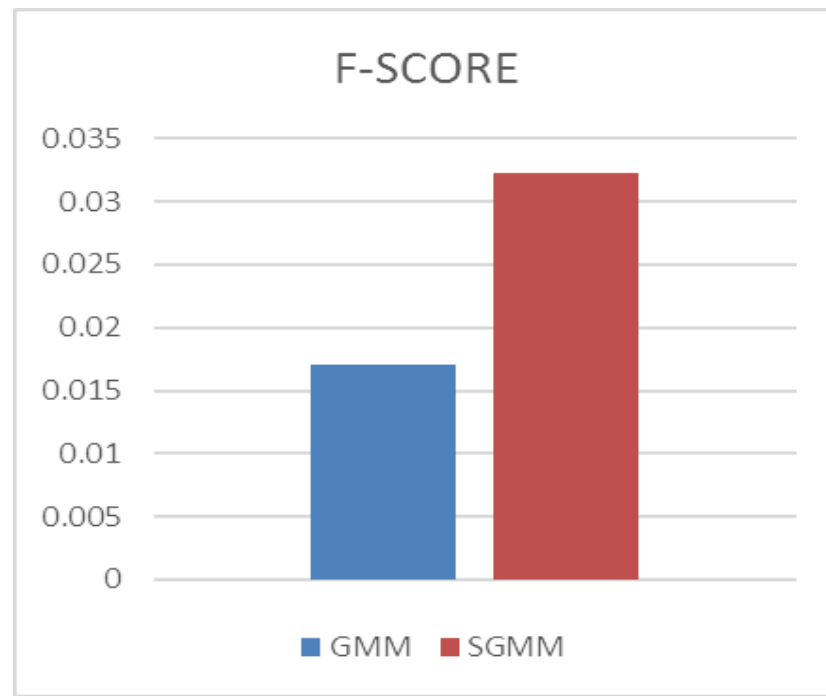

Fig.25 F-Score of proposed method on dynamic back ground dataset

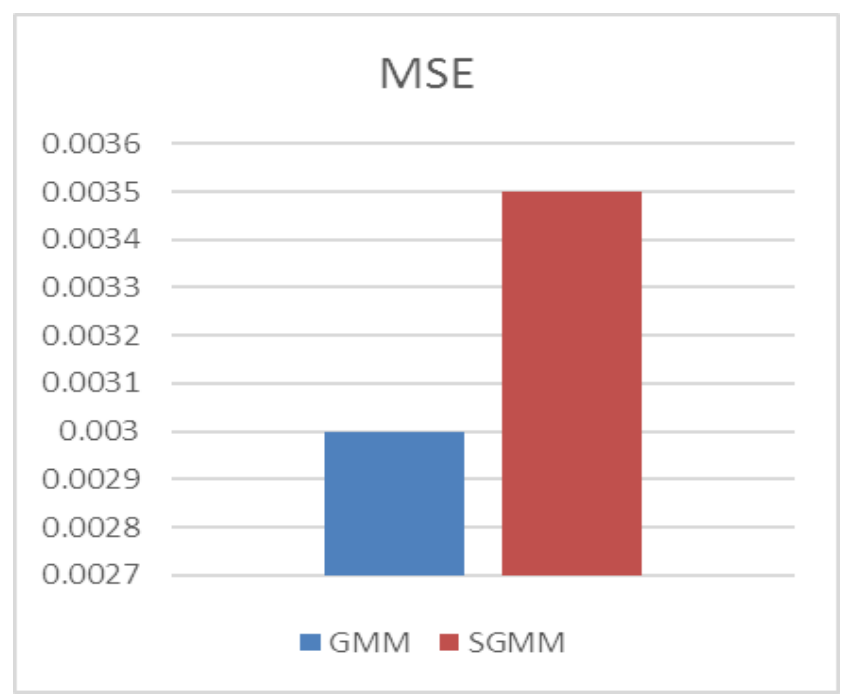

Fig.26 MSE of proposed method on dynamic back ground dataset

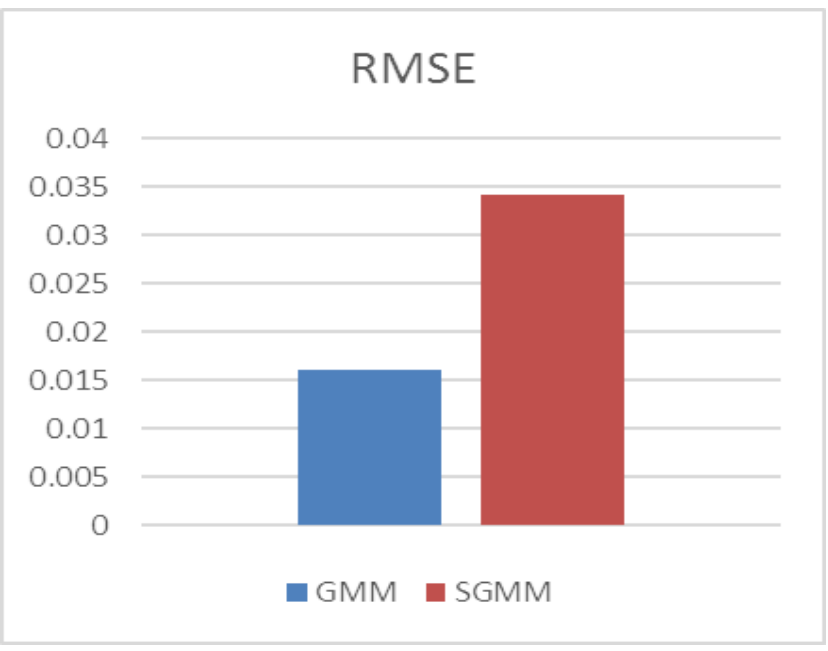

Fig.27 RMSE of proposed method on dynamic back ground dataset

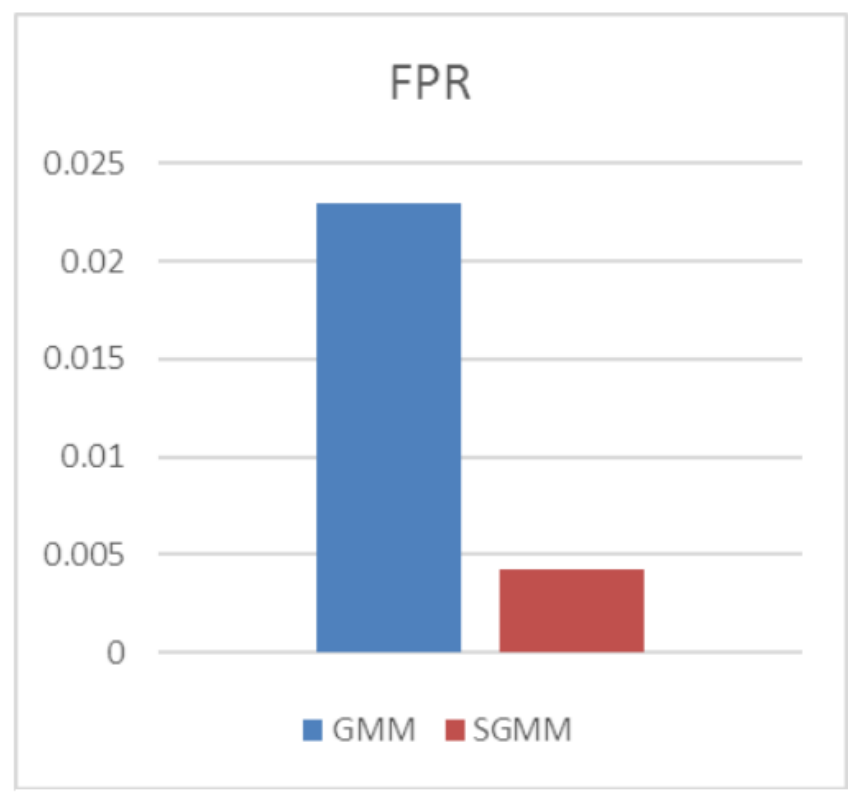

Fig.28 FPR of proposed method on dynamic back ground dataset

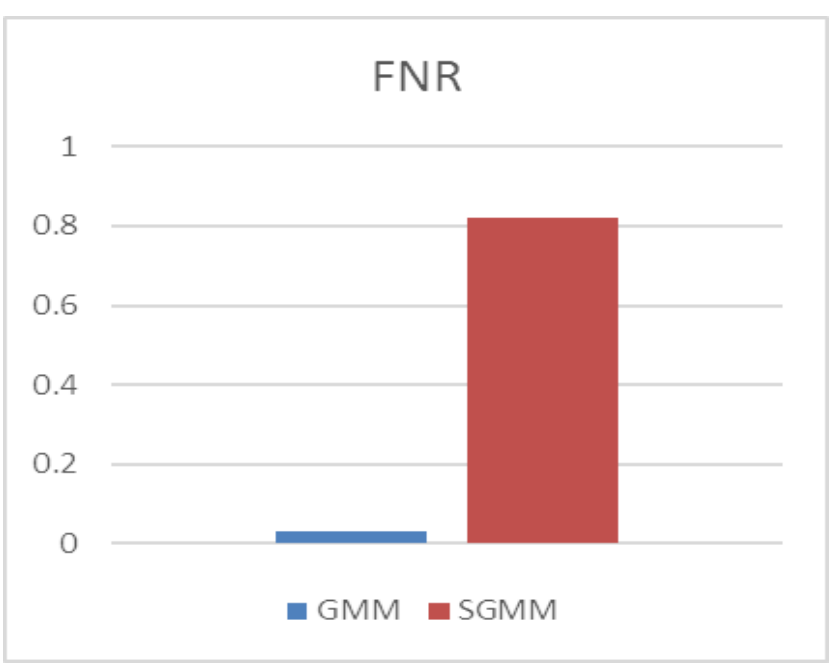

Fig.29 FNR of proposed method on dynamic back ground dataset

Published By:

1742 Blue Eyes Intelligence Engineering

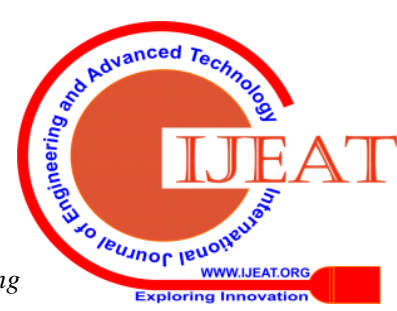




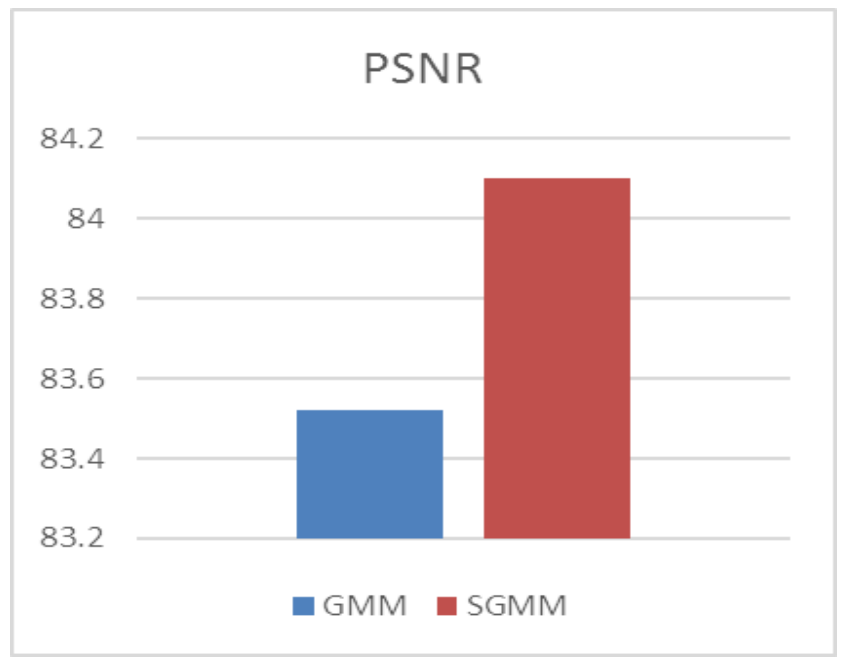

Fig.30 PSNR of proposed method on dynamic back ground dataset

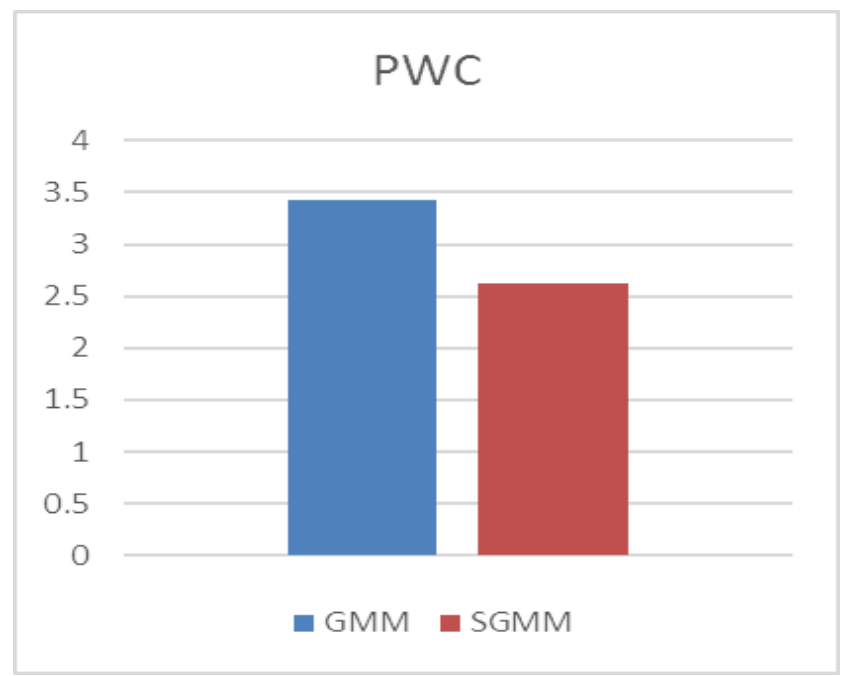

Fig.31 PWC of proposed method on dynamic back ground dataset

TABLE .4

\begin{tabular}{|l|r|r|}
\hline \multicolumn{3}{|c|}{$\begin{array}{c}\text { Evaluation Metrics of different methods on } \\
\text { SHADOW video from CD net DATASET }\end{array}$} \\
\hline $\begin{array}{c}\text { Metrics } \\
\text { Methods }\end{array}$ & GMM & SGMM \\
\hline PRECISION & 0.0232 & 0.0532 \\
\hline RECALL & 0.0132 & 0.0587 \\
\hline ACCURACY & 0.9448 & 0.9932 \\
\hline F-SCORE & 0.0132 & 0.0182 \\
\hline MSE & 0.0367 & 0.0072 \\
\hline RMSE & 0.0040 & 0.0902 \\
\hline FPR & 0.0132 & 0.0182 \\
\hline FNR & 0.9325 & 0.9642 \\
\hline PSNR & 73.2872 & 85.262 \\
\hline PWC & 3.0023 & 2.6055 \\
\hline
\end{tabular}

Fig.33 Recall of proposed method on Shadow dataset

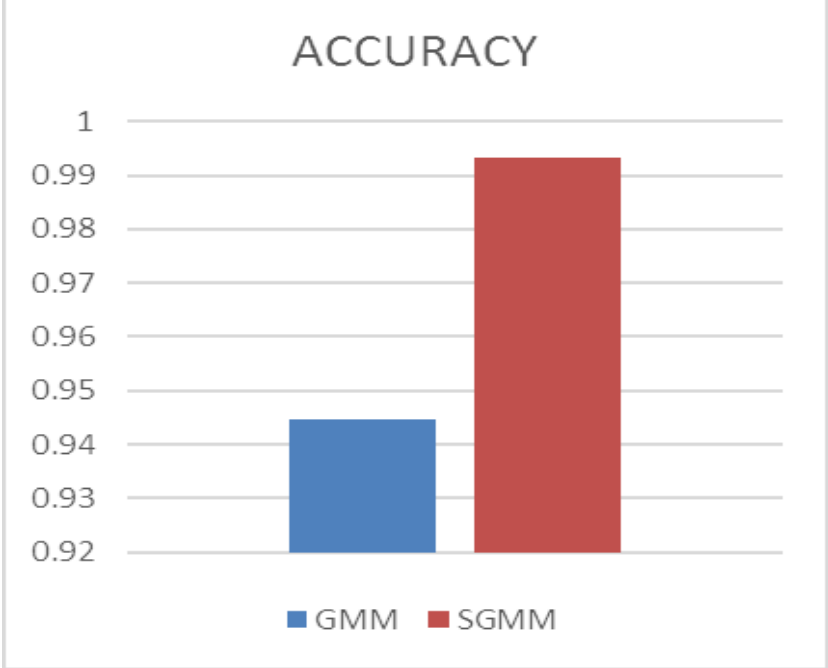

Fig.34 Accuracy of proposed method on Shadow dataset 
Towards the Development of Effective Video Segmentation Based on Skew Gaussian Mixture Model

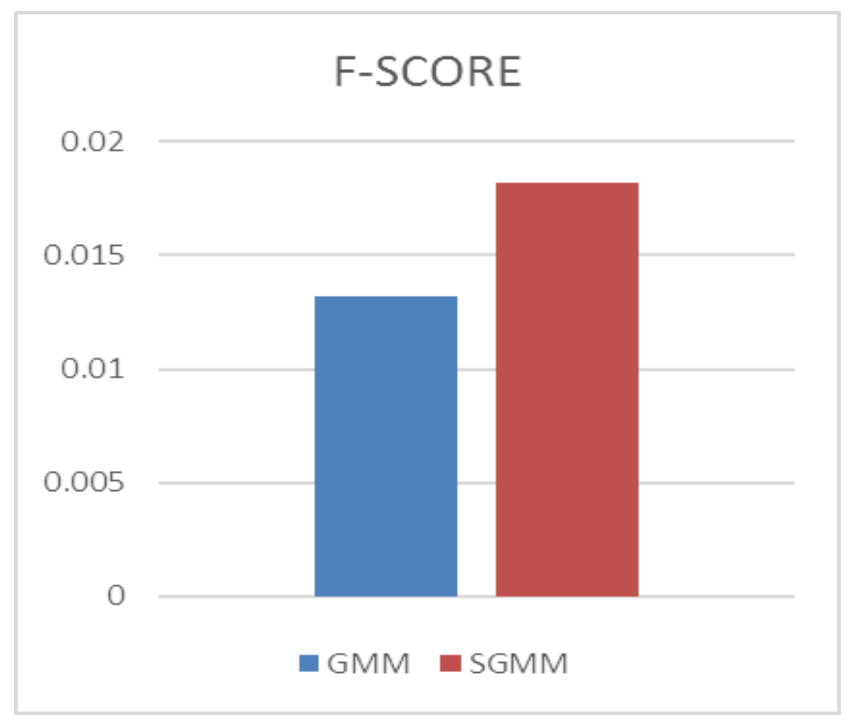

Fig.35 F-Score of proposed method on Shadow dataset

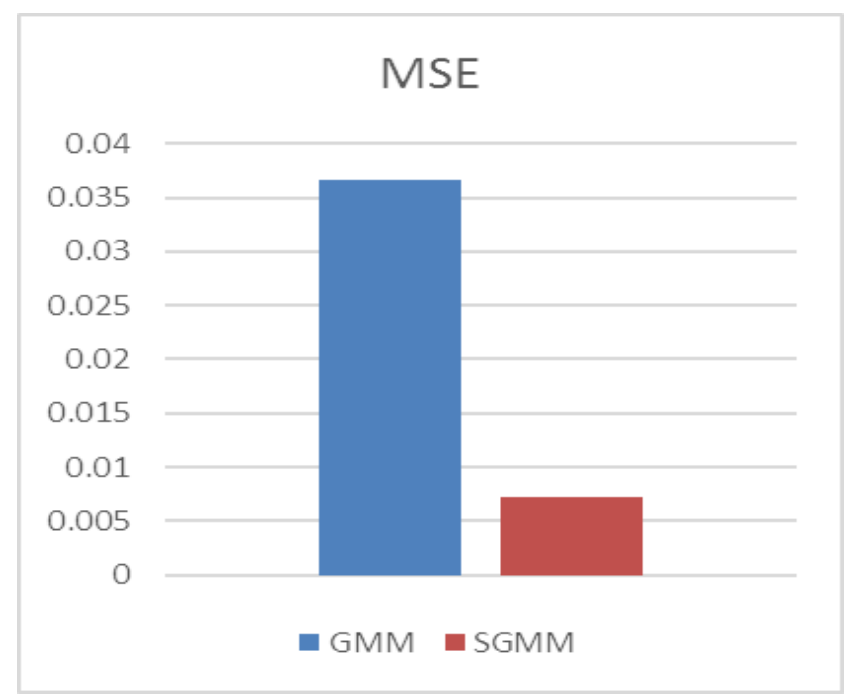

Fig.36 MSE of proposed method on Shadow dataset

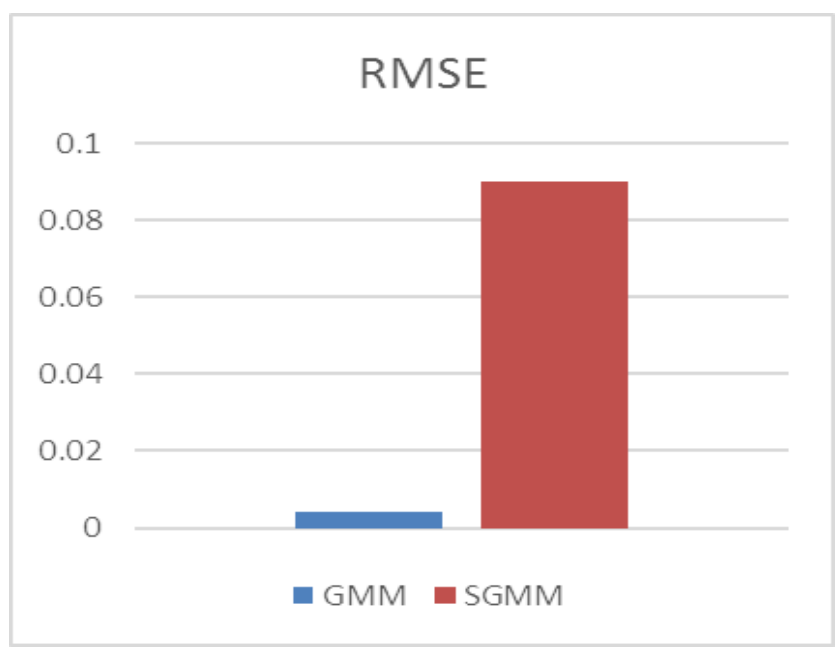

Fig.37 RMSE of proposed method on Shadow dataset

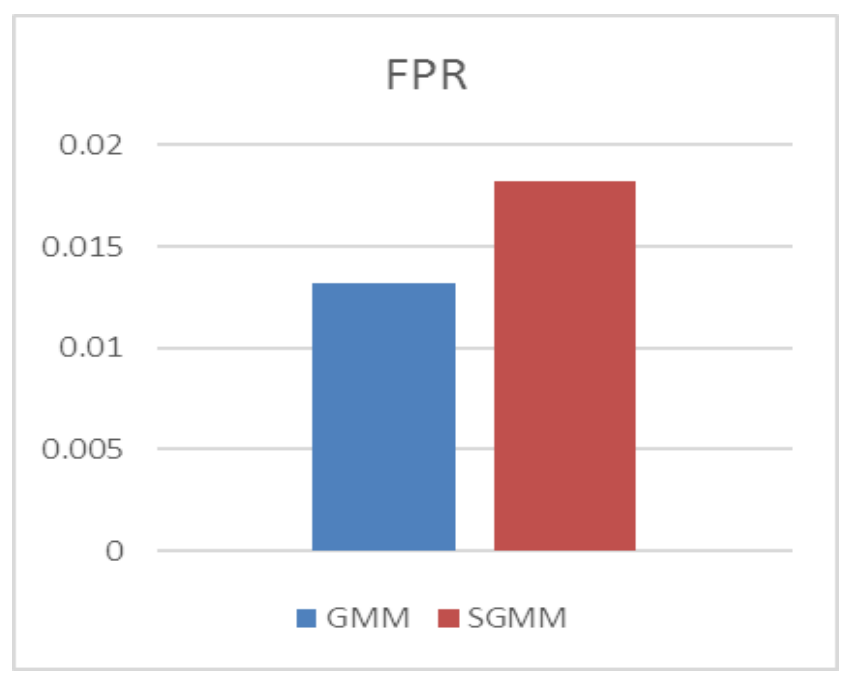

Fig.38 FPR of proposed method on Shadow dataset

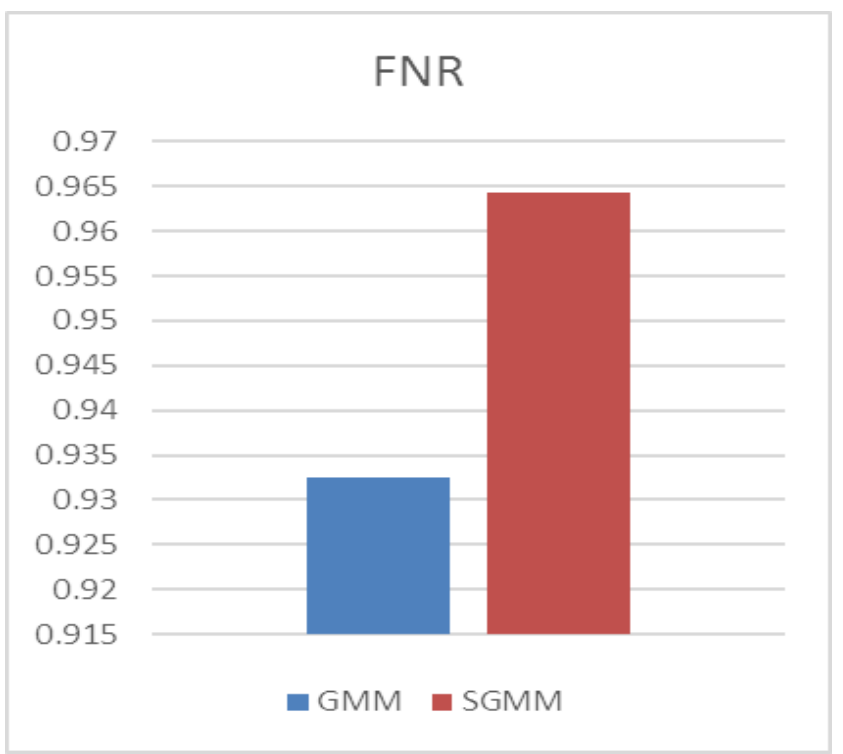

Fig.39 FNR of proposed method on Shadow dataset

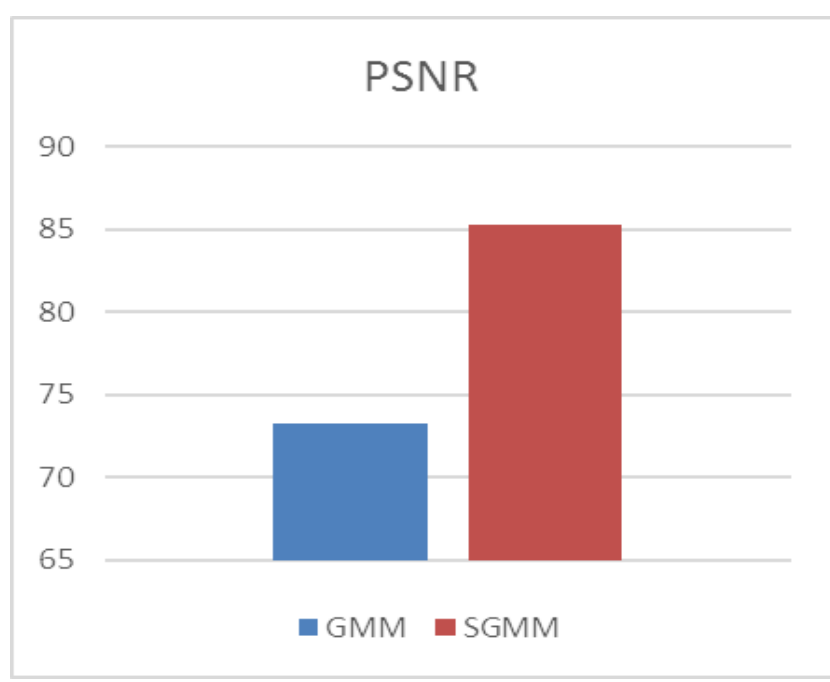

Fig.40 PSNR of proposed method on Shadow dataset 


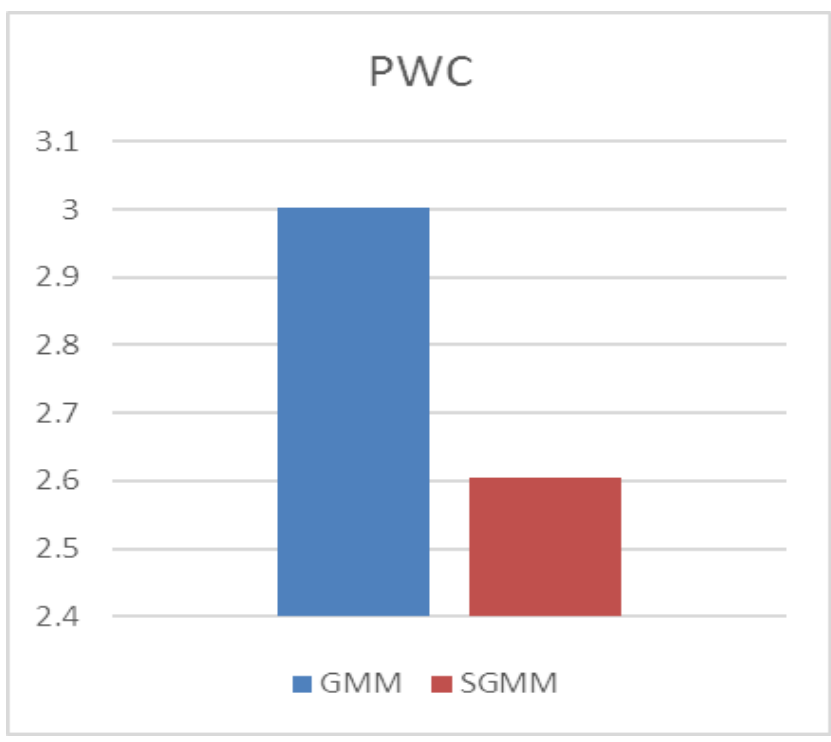

Fig.41 PWC of proposed method on Shadow dataset

\section{VII.CONCLUSION}

In this article, a model proposed for the effective segmentation of images based on the background information .The initial values of parameters are processed to obtain the convergent values using E.M alogorithm. The experimentation results derived and also it is compared with data of the existing models based on Gaussian Distribution using the performance metrics like FPR, FNR, F-score, precision, Recall, etc. Specified in table -1 to table -4 and figures 2-41.From the above information presented in the tables and graphs it can be clearly identify that the proposed methodology perform well with respect to all the parameters and the results. The proposed methodology can be very well suited for most of the applications pertaing to segmentation.

\section{REFERENCES}

1. Michael Unger, Mark Asbach, and Peter Hosten "Enhanced background subtraction using global motion compensation and mosaicing", 978-1-4244-1764-3/08/ IEEE pp.2708-2711, 2008.

2. Nagesh Vadaparthi, Srinivas Y, P Suresh Varma, P S Sitharama Raju, "Hierarchical Clustering technique for Medical Image Segmentation Based on Finite Skew Gaussian Mixture Model and EM Algorithm", The International Arab Journal of Information Technology, 15(5), September 2016

3. Pavan Kumar T, Srinivas Y, Nagesh Vadaparthi, “A Novel Approach for Background Subtraction using Generalized Rayleigh Distribution", (IJACSA) International Journal of Advanced Computer Science and Applications, Vol. 9, No. 9, 2018

4. A.Monnet, AMittal, N.Paragios, V.Ramesh, Background modeling and subtraction of dynamic scenes, in: proceedings of IEEE International conference on computer vision, 2003, pp.1305-1312

5. Z.Zivkovic, Improved adaptive Gaussian mixture model for background subtraction in: proc. $17^{\text {th }}$ International conference pattern Recognit., vol.2.Aug.2004. pp.28-31

6. H.Zhov, Y.Chen, and R.Feng, " A novel background subtraction method based on color invariants. "computer vis. Image understand vol. 117, no.11, pp.1589-1597, nov.2013

7. A.Akula, NKhanna, R.Ghosh, S.kumar, A.Das and H.k Sardana , “ Adaptive contour-based statistical background subtraction Method for moving target detection in infrared video sequences, "Infr. Phys Technol., vol.63, pp.103-109, mar.2014

8. A.Viswanth, R.Kumari behra, V.Scnthamilarasu, K.Kutty, "Background modeling from a moving camera." Elsevier second international symposium on computer vision and the internet vol.58, pp.289-296, 2015
9. Weiweri D, Yujian W, Wenpeng J, Taoxin P, 'An improved Gaussian mixture model method for moving object detection. Telecommunication, Computing electronics and control (TELKOMINIKA)2016.14 (13) - 115-123

10. S.Kanagamalliga, S.Vasuki, M.Shanmugapriya " Foreground object detetion using Expectation maximization based effective Gaussian mixture model" Innovations in information, Embedded and communication systems, issues 24, pp.51-57-2016

11. Yong XU, J.Dong, B.Jhong, D.XU, "Background modelling methods in video analysis : A Review and comparative evaluation" CAAI Transaction an Intelligence Technology 1, pp 43-60, 2016

12. Satis kumar N, Shobha G "Background modeling to Detect Foreground objects based on ANN and Spatio- temporal Analysis. Indonesian Journal of electrical engineering and computer science. 2017; 15(4) :151-160

13. L.Vosters, C.Shan, and T.Gritti, "Background subtraction under sudden illumination changes." In proc IEEE Int. conference Advanced video signal based surveillance, Aug.2010, pp.384-391

14. Bouwmans T, EI-BatF, Vachan B (2010) Statistical background modeling for foreground detections: a survey. In: Hand book of pattern recognition and computer vision, 4(2), world scientific publishing, pp181-199.

15. Spagnolo, Paolo, M.Leo and A.Distante "Moving object segmentation by background subtraction and temporal analysis." Image and vision computing 24.5, pp. 411-423, 2006

\section{AUTHORS PROFILE}

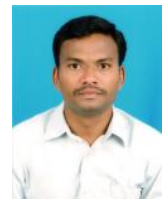

PavanKumar Tadiparthi, is working as a Associate Professor in the department of information Technology, MVGR College of Engineering, Vizianagram, Andhra Pradesh, india.He has about 13 years of teaching experience and his area of interest is image processing. $\mathrm{He}$ is a life member of ISTE, MIE

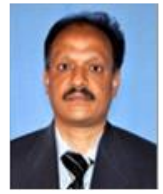

Srinivas Yarramalle, is a Professor in the department of Information Technology, GITAM India and has about 23 years of teaching experience GITAM University, and Visakhapatnam. His areas of interests include Speech Processing, Data mining, and Software reusability apart from Image processing. He is having more than 200 publications at national and International level. He is the author for 4 books and a life member of ISTE, CSI, IE, ISTAM, IISA and ISPS. 